Meta

Journal des traducteurs

Translators' Journal

\title{
Le Dictionnaire historique de la langue hébraïque
}

\section{Israël Yevin}

Volume 43, numéro 1, mars 1998

La traduction et l'interprétation en Israël

Translation and Interpreting in Israel

URI : https://id.erudit.org/iderudit/004632ar

DOI : https://doi.org/10.7202/004632ar

Aller au sommaire du numéro

Éditeur(s)

Les Presses de l'Université de Montréal

ISSN

0026-0452 (imprimé)

1492-1421 (numérique)

Découvrir la revue

Citer cet article

Yevin, I. (1998). Le Dictionnaire historique de la langue hébraïque. Meta, 43(1), 19-26. https://doi.org/10.7202/004632ar

\section{Résumé de l'article}

En 1959, l'Académie de la langue hébraïque a entrepris la rédaction d'un grand Dictionnaire historique de la langue hébraïque retraçant l'histoire du vocabulaire hébraïque, depuis les premières attestations écrites (vers -200 avant J.-C.) jusqu'à nos jours. En plus de la description historique (changements d'acceptions, d'emplois et de formes des mots au cours des âges), le dictionnaire est appelé à comporter des rubriques étymologiques, des définitions, des marqueurs sémantiques et une rubrique bibliographique. 


\title{
LE DICTIONNAIRE HISTORIQUE DE LA LANGUE HEBRAIIQUE
}

ISRAËL YEVIN

Université hébraïque de Jérusalem et Académie de la langue hébraïque,

Jérusalem, Israël

\begin{abstract}
Résumé
En 1959, l'Académie de la langue hébräque a entrepris la rédaction d'un grand Dictionnaire historique de la langue hébraïque retraçant l'histoire du vocabulaire hébraïque, depuis les premières attestations écrites (vers -200 avant J.-C.) jusqu'à nos jours. En plus de la description historique (changements d'acceptions, d'emplois et de formes des mots au cours des âges), le dictionnaire est appelé à comporter des rubriques étymologiques, des définitions, des marqueurs sémantiques et une rubrique bibliographique.
\end{abstract}

\begin{abstract}
The history of the Hebrew language spans approximately three thousand years. However, the only historical dictionary currently available, Eliézer Ben-Yehouda's Thesaurus was published some ninety years ago before the discovery of numerous manuscripts and before the advances in scientific research. In 1959, the Hebrew Language Academy began working on the Historical Dictionary of the Hebrew Language, tracing the history of Hebrew vocabulary from its first written attestations to present day. Beyond the historical description it contains, covering changes in meanings, usage and form of words, the dictionary will provide sections on etymology, definitions, semantic markers and a bibliography.
\end{abstract}

\section{POURQUOI UN DICTIONNAIRE HISTORIQUE ?}

L'histoire de la langue hébraïque, depuis ses origines jusqu'à nos jours, couvre approximativement trois mille ans. Les manuscrits et textes exploitables les plus anciens remontent aux environs de l'an 200 avant J.-C. Or toute langue vivante, on le sait, ne cesse d'évoluer. Les mots changent d'acception. Ainsi, en hébreu biblique, le substantif aviv désigna d'abord «les produits agricoles avant leur mûrissement» et signifie aujourd'hui «printemps». Autrefois néchef désignait «le soir»; il désigne aujourd'hui un «bal», une «fête», une «soirée». La langue évolue également au niveau morphologique. C'est ainsi que le démonstratif pluriel était élé, dans l'hébreu biblique, alors que dans l'hébreu michnique, il devint élou. Dieu a d'abord été caractérisé par cette locution nominale : Hakodèch baroukh hou («la sainteté, bénie soit-elle») avant d'être qualifié par une forme adjective : Hakadoch baroukh hou («le saint, béni soit-il»). Certains mots sont abandonnés et remplacés par d'autres : la première personne du pronom personnel fut d'abord anokhi, remplacée par la suite par ani («je»); le pronom relatif est acher, en hébreu biblique, et devient un préfixe, chè-, dans l'hébreu talmudique, etc. D'où la nécessité d'un dictionnaire historique qui rende compte de toutes ces transformations.

On peut établir un parallélisme entre dictionnaire historique et grammaire historique. On distingue, en effet, la grammaire synchronique (qui décrit l'état actuel de la phonologie et de la morphologie d'une langue donnée) et la grammaire historique (qui retrace l'histoire des systèmes phonologiques et morphologiques et leurs transformations, au fil des âges). La même distinction vaut pour les dictionnaires. Ces derniers peuvent être syn- 
chroniques, comme le Dictionnaire hébrä̈que d'Abraham Even-Chochan (7 vol., KiryatSéfer, Jérusalem, 1966-1970), qui décrit l'hébreu israélien, dans son usage littéraire et parlé. Mais il peuvent aussi être diachroniques et décrire alors l'évolution de chaque mot au sein de la langue : son origine, son étymologie, son mode de dérivation, mais aussi ses acceptions, ses emplois dans des locutions aux diverses époques du développement de la langue, ses variantes morphologiques (tant sur le plan de l'orthographe et de la vocalisation que sur le plan grammatical), enfin sa fréquence aux diverses époques considérées. La plupart des pays de cultures anciennes, à travers le monde, ont entrepris d'élaborer un dictionnaire historique de leur langue nationale. Certains se concentrent sur les langues vivantes (comme le Oxford English Dictionary), d'autres décrivent des langues qui ont cessé d'être parlées (comme le Middle English Dictionary).

Pour l'hébreu, nous ne possédons pratiquement qu'un seul dictionnaire historique : celui d'Eliézer Ben-Yehouda (Thesaurus de la langue hébrä̈que ancienne et moderne, 17 vol., Berlin, Langenscheidt, 1910-1959). Bien qu'étant l'œuvre d'un seul homme, il contient une quantité énorme d'attestations de toutes les périodes de l'hébreu et il fait couramment appel aux sources exégétiques et philologiques, lexicologiques et grammaticales de l'hébreu ancien, sans négliger la littérature scientifique moderne et les manuscrits, y compris les variantes existantes et les fragments hébraïques retrouvés dans des guenizoth (pièces où l'on mettait au rebut les manuscrits inutilisables).

Mais depuis la parution du premier volume de cet ouvrage, il y aura bientôt quatrevingt-dix ans, bien des choses ont changé. Des progrès considérables ont été réalisés par la recherche scientifique, qui a étudié les diverses époques de l'hébreu, plus particulièrement l'hébreu de l'époque talmudique, appelé lechone 'hakhamim («la langue des Sages», qui désigne en fait l'hébreu «michnique»). L'enseignement des rabbins de l'Antiquité est en effet recueilli dans le Talmud, vaste corpus constitué de la Michna, compilée au deuxième siècle et rédigée en hébreu, tandis que la Guemara, rédigée en araméen, est plus tardive et représente essentiellement un développement de la Michna. Les progrès de la linguistique historique ont été favorisés par le développement de la manuscriptologie et par la découverte de nouveaux documents et de fragments d'œuvres inconnues auparavant : fragments de midrachim (exégèse homilétique, à caractère moral ou légendaire), poèmes liturgiques, etc. La découverte des Manuscrits de la mer Morte, dans le désert de Judée (entre 1947 et 1956) et le déchiffrement des fragments de la Gueniza du Caire (mise au jour en 1896, mais étudiée activement ces dernières décennies) ont enrichi le corpus hébraïque, mais ils ne figurent évidemment pas dans le thésaurus d'Eliézer Ben-Yehouda.

C'est pourquoi, en 1959, l'Académie de la langue hébraïque a décidé d'entreprendre la rédaction d'un grand Dictionnaire historique de la langue hébraïque retraçant l'histoire du vocabulaire hébraïque depuis ses origines jusqu'à nos jours et se fondant sur le traitement et l'examen de milliers de sources écrites, depuis l'apparition de l'hébreu jusqu'à nos jours. En plus de comporter une description historique (c'est-à-dire les changements d'acceptions, d'emplois et de formes des mots au fil des âges), le dictionnaire doit comprendre des rubriques étymologiques, des définitions, des marqueurs sémantiques et une rubrique bibliographique. L'ouvrage met à profit les attestations (écrites et orales) de la morphologie et de la prononciation hébraïques : transcriptions, traditions de prononciation conservées dans les diverses communautés juives, témoignages de manuscrits vocalisés (rappelons que l'hébreu ne note que les consonnes, de sorte que la plupart des manuscrits ne comportent pas de voyelles). 


\section{LE PROGRAMME DE DICTIONNAIRE HISTORIQUE DE L'ACADÉMIE}

\subsection{Les principes directeurs du projet}

Le Dictionnaire historique de la langue hébräque est un programme de recherche de l'Académie de la langue hébraïque. L'initiative en revient au professeur Zeev Ben'Haïm, qui en fut le premier directeur et rédacteur en chef. Le directeur et rédacteur actuel est le professeur Avraham Tal, secondé par une équipe scientifique d'une vingtaine de personnes.

\subsubsection{La datation des textes cités}

L'un des principes fondamentaux, dans tout dictionnaire historique, est la datation de chaque citation et son classement chronologique. Chaque fiche établie pour une citation doit donc porter une mention de date aussi précise que possible. Ainsi, toutes les ouvres rédigées avant l'invention de l'imprimerie sont citées à partir des manuscrits, et non pas de leur édition ultérieure sous forme de volume imprimé, même lorsque ces éditions sont devenues classiques (comme l'édition Vilna, pour le Talmud) ou qu'il s'agit d'éditions scientifiques. Même dans ces cas, on s'appuie uniquement sur les manuscrits les plus anciens et les mieux conservés. Chaque citation du dictionnaire est donc accompagnée de deux dates : la date de rédaction de la citation et la date (présumée ou attestée) du manuscrit dans lequel elle figure. En ce qui concerne la littérature hébraïque ancienne, l'écart entre ces deux dates peut être parfois considérable. C'est ainsi que le Midrach Sifra (exégèse sur le Lévitique) a été rédigé avant l'an 300 de l'ère vulgaire, le manuscrit Vitkin date de l'an 66, mais le manuscrit le plus fiable, dont on se sert pour les citations de Sifra, est l'œuvre d'un copiste de l'an 950 environ. Pour le Talmud, on sait par exemple que le traité Zeva'him («Les sacrifices») a été rédigé autour de l'an 500, tandis que le manuscrit Colombie (qui fait autorité pour ce traité) a été copié en 1546. Quant aux œuvres rédigées après l'invention de l'imprimerie, on les traite à partir de l'édition princeps ou de leur première apparition imprimée. C'est ainsi, par exemple, qu'un article du penseur sioniste A'had Haam (1856-1927), publié d'abord dans le mensuel hébraïque Hachiloa'h et intégré, par la suite, dans son recueil d'essais Al Parachate Derakhim («À la croisée des chemins», 1905, édition définitive en 1922, édition critique en 1957-1960), sera cité d'après la date de sa première publication dans Hachiloa'h.

\subsubsection{Le retour aux manuscrits}

Comme on le sait aujourd'hui, grâce notamment aux recherches effectuées sur l'hébreu michnique, l'examen des manuscrits est particulièrement important pour la connaissance de la langue des sources anciennes. Les textes imprimés ont souvent supprimé de nombreux traits originaux de l'hébreu de l'Antiquité en se livrant à un travail de standardisation et d'uniformisation destiné à donner une forme et un aspect aussi réguliers que possible à toutes les couches de la langue. Le traitement des sources, à partir des manuscrits les mieux conservés, est susceptible de nous révéler une image bien plus exacte des mots, de leur acception, de leur emploi à chaque époque du développement de l'hébreu. C'est pourquoi le Dictionnaire historique s'est donné pour principe fondamental de ne traiter les sources anciennes qu'à partir des manuscrits. Et c'est l'un des traits qui distingue le dictionnaire de l'Académie des autres dictionnaires et des concordances établis pour l'hébreu ancien. À titre d'exemple, les éditions imprimées (et donc les dictionnaires et les concordances qui s'appuient sur ces éditions) gomment la différence entre les variantes suivantes : Ate/ata («tu», «toi», pronom personnel; deux formes attestées de la deuxième personne du masculin), halalou/haèlou («ceux-ci»; deux formes attestées du démonstratif pluriel), hitpaèl/nitpaël (changement morphologique du paradigme du verbe pronominal), bate'hila/kate'hila («au début»; changement de préposition préfixée), mataï/eymataï 
(«quand ?»), heymènou/mimènou («de lui»). Jusqu'à présent, ces différences — essentielles pour reconstituer l'évolution de la langue — étaient estompées, supprimées par volonté d'harmonisation, au point qu'il était impossible de pratiquer une étude linguistique sérieuse en se fondant sur les éditions imprimées et les dictionnaires et concordances qui en découlent. Seule l'étude des manuscrits permet de mettre au jour le statut et l'emploi de chacune de ces variantes.

\subsubsection{Le recours à l'informatique}

Un autre grand principe, dans la préparation du Dictionnaire historique, est le recours à l'informatique. Aujourd'hui, la chose semble aller de soi. Mais ce choix était révolutionnaire lorsque l'Académie a entrepris son projet, en 1959. L'informatique était alors peu utilisée en lexicologie, à travers le monde, et il faut rendre hommage à l'audace du professeur Ben-'Haïm, concepteur du projet ${ }^{1}$.

L'usage de l'outil informatique présente quatre avantages majeurs :

a) l'ordinateur est particulièrement efficace dans le traitement du matériel. Comparé au traitement manuel, il économise un temps considérable dans la préparation de la concordance d'un texte;

b) seul l'ordinateur permet de stocker commodément de grandes quantités de matériel : des millions de mots, accompagnés de leur contexte, de leur analyse, d'une référence, etc., comme il convient à un dictionnaire historique;

c) l'ordinateur permet une mise à jour régulière et permanente du matériel stocké. Une fois achevé le traitement du matériel pour une période donnée, il se peut qu'une œuvre nouvelle soit découverte qui mérite d'être intégrée au corpus de cette période. Il faut la traiter et inclure chacun de ses mots à l'intérieur du corpus déjà élaboré. Grâce à l'ordinateur, c'est un jeu d'enfant. On peut de même introduire, sans difficultés et très rapidement, toute correction, tout changement dans la compréhension d'un terme, dans sa lecture, dans son analyse grammaticale;

d) l'ordinateur permet enfin (et c'est l'un de ses avantages essentiels) de traiter le matériel sous des angles différents. Prenons l'exemple du traitement grammatical. L'ordinateur peut extraire du corpus toutes les formes stockées appartenant à une même racine : un paradigme verbal et sa déclinaison, la morphologie d'un nom et ses conjugaisons, etc. Il peut aussi fournir des listes lexicologiques indiquant notamment la fréquence des mots et une comparaison de cette fréquence en fonction de l'époque considérée. Il peut également procurer des listes de combinaisons possibles d'un mot avec d'autres mots de la langue et vérifier leur fréquence afin de faire ressortir les collocations, les mots composés, les locutions, les mots appelés impérativement par un verbe, etc.

\subsection{Les périodes traitées}

Le programme de dictionnaire historique s'occupe actuellement de traiter exclusivement deux périodes de l'histoire de l'hébreu : la période ancienne, depuis la clôture du canon biblique jusqu'à la fin de l'époque des Gaonim (nom donné aux chefs de la communauté de Babylonie, démantelée aux environs de l'an 1050), et la période moderne (1750 à nos jours). Pour ces deux périodes, l'Académie a déjà publié les sources existantes. En voici les références, pour l'hébreu ancien : Sefer hamekoroth, 'helek richone («Livre des Sources, première partie; de la clôture du canon biblique à la fin de l'ère gaonique», Jérusalem, 1963-1970). Le volume contient la liste de toutes les œuvres rédigées à l'époque ancienne, des manuscrits qui les renferment et de leurs éditions scientifiques). Pour la période moderne, un premier fascicule a été publié : Sefer hamekoroth litekoufa chèmine 
1750 veilakh («Livre des Sources, à partir de 1750; premier fascicule : choix d'œuvres dans les Belles Lettres hébraïques, 1860-1920», Jérusalem, 1987).

En ce qui concerne la période 1050-1750, un modus operandi a été élaboré, il y a déjà quelques années, pour l'examen de la vaste littérature hébraïque de cette époque. Elle sera étudiée par blocs, en distinguant les catégories littéraires et les lieux géographiques d'origine. Le travail a déjà commencé pour la littérature rabbinique d'Afrique du Nord, depuis l'élaboration de ses formes nouvelles, à la fin du XIV ${ }^{\mathrm{e}}$ siècle, à la suite des persécutions de 1391, lorsque trois rabbins éminents vinrent s'installer en Afrique du Nord : Rabbi Yits'hak Bar Chichat (le Ribach), Rabbi Chimon Bar Tséma'h (le Rachbats) et Rabbi Ephraïm Ankaoua. Les chercheurs ont déjà préparé un «Livre des Sources» pour les années 1391-1960, qui comprend l'ensemble de la littérature rabbinique imprimée, classée par catégories (y compris la poésie liturgique et les chansons), ainsi que des œuvres diverses : chroniques, inscriptions tumulaires, plaques dédicatoires, etc. (La parution est prévue en 1998.)

\subsection{Le traitement du corpus}

Les œuvres des périodes anciennes ne constituent qu'un vestige d'une certaine phase de la vie de la langue hébraïque. Elles l'éclairent partiellement et jouissent d'un statut particulier du fait de leur influence sur l'évolution de la langue hébraïque des époques ultérieures, y compris de notre époque. Elles méritent donc un traitement intégral, c'est-à-dire l'établissement de concordances exhaustives, fondées sur les manuscrits les mieux conservés. Le traitement sous forme de concordance exhaustive est «objectif» puisqu'il n'est pas influencé par les considérations personnelles du chercheur, qui déciderait quel mot inclure et quel mot négliger. Grâce à l'informatique, ce traitement est également beaucoup plus simple et rapide aujourd'hui qu'il ne l'était autrefois. C'est pourquoi le comité de rédaction du dictionnaire a choisi de traiter la totalité des œuvres connues de la période ancienne sous forme de concordance intégrale. Par contre, l'abondance des œuvres des périodes postérieures empêche l'établissement de concordances exhaustives. Il faut bien se résoudre à faire un choix dans le matériel à traiter en vue de la préparation du dictionnaire, faute de quoi le travail risquerait de durer indéfiniment. Mais le problème de la sélection s'avère complexe : quelle source privilégier, quel texte rejeter ? Il faut faire appel à des critères rigoureux et faire preuve de discernement.

\subsubsection{Volume des sources traitées}

Pour la littérature ancienne, le corpus traité sous forme de concordances portera sur près de sept millions de mots, ainsi répartis selon les catégories littéraires auxquelles ils appartiennent :

Rouleaux du désert de Judée et inscriptions

Littérature des Tannaïm, Talmud et Midrach

3600000

Époque des Gaonim

1150000

Textes caraïtes

Poésie liturgique

980000

Écrits des Massorètes et des grammairiens

150000

Total

6550000

L'essentiel du matériel a déjà été traité et il ne reste plus qu'à mener à bien les dernières phases du travail pour pouvoir publier, prochainement, la concordance relative à cette période. 
En ce qui concerne la littérature moderne et contemporaine (depuis le milieu du XVIII ${ }^{\mathrm{e}}$ siècle), les œuvres sélectionnées (plus de cinq cents) exigent le traitement de plus de huit millions de mots appartenant aux catégories suivantes : belles-lettres (tous les genres); presse (articles, essais, feuilletons, polémiques, fragments journalistiques); œuvres de moralistes et littérature 'hassidique; sciences naturelles et médecine; histoire; géographie et chroniques de voyageurs; biographies; correspondance et recueils d'épîtres; livres de coutumes et usages religieux; préfaces de dictionnaires, de grammaires, de romans et poèmes, de livres de prière. Le traitement des sources est pratiquement achevé et près de la moitié du travail de préparation de concordances lexicographiques a déjà été effectuée. Le traitement de la seconde moitié est en cours.

\subsection{2. Échantillons disponibles}

Jusqu'à ce jour, le travail de l'équipe du Dictionnaire historique a consisté essentiellement à réunir le matériel et à recueillir les témoignages attestant des divers états de la langue. Mais le traitement du corpus et son élaboration dans le format d'un dictionnaire n'a pas véritablement commencé, sinon sous forme d'échantillons. C'est ainsi qu'en 1982, l'Académie a publié, dans sa revue Lechonènou, un exemplaire de la façon dont se présentera, dans le Dictionnaire historique, le traitement d'une racine hébraïque et de tous ses dérivés (Zeev Ben-'Haïm, «La racine ayine/rèch/beyt», Lechonènou, vol. 46, 3-4, 1982, pp. 165-267). Sur 11000 citations comprenant la racine 'RV, 1400 ont été retenues pour illustrer les 102 lemmes dérivant de cette racine. Quant à l'équipe de la section «Littérature moderne», elle achève actuellement un premier échantillon de dictionnaire stylistique. Il porte sur le vocabulaire hébraïque des œuvres du grand romancier Mendele Mokher Seforim (1835-1917), l'un des premiers écrivains yiddish à avoir adhéré à la Renaissance littéraire hébraïque en Russie. Ce dictionnaire stylistique comprendra 1050000 entrées.

\subsubsection{L'archivage des sources}

Dans l'intervalle, des quantités considérables de témoignages écrits s'accumulent dans les bureaux du «Programme de Dictionnaire historique». Ce matériel est recueilli de manière objective, c'est-à-dire qu'il est exhaustivement traité sans qu'intervienne le moindre élément subjectif. Sa mise en œuvre répond à des critères scientifiques minutieux : lecture scrupuleuse de la version du manuscrit considéré et restitution exacte, analyse des mots en fonction de l'exégèse et de la littérature scientifique la plus récente; possibilité permanente de mise à jour. Cette collecte est en soi d'une importance extrême. Ses dimensions sont plus vastes que celles du futur dictionnaire. Entièrement informatisé, le matériel est à même d'être exploité de toutes les manières possibles. De fait, il constitue en quelque sorte des archives de la langue hébraïque, comportant une quantité considérable de matériel exact, attesté et précis sur l'histoire de la langue hébraïque. Ce corpus servira à la rédaction du Dictionnaire historique, mais il pourra également donner lieu à des recherches ou à divers travaux, tels qu'une vérification de certains phénomènes grammaticaux, sémantiques et autres; une étude descriptive de la langue propre à un certain document; les caractéristiques de la langue d'une certaine époque, etc. Il s'agit d'archives permanentes qui ne cessent d'accueillir de nouveaux matériaux et de se mettre à jour. Elles sont d'ores et déjà à la disposition des chercheurs et des personnes intéressées. Si la rédaction du Dictionnaire historique reste l'objectif ultime et le couronnement du programme de recherche qui porte son nom, il faut reconnaître qu'il ne sera mené à bien qu'au terme de nombreuses années de travail supplémentaire, tandis que les archives permanentes de la langue hébraïque ont déjà vu le jour. L'équipe rédactionnelle du dictionnaire a déjà exploité ces archives en publiant une concordance intégrale, fondée sur 820000 mots classés autour de leurs racines primordiales, de toutes les œuvres hébraïques postbibliques, rédigées jusqu'à la fin 
de l'époque des Tannaïm (les maîtres de la Michna, du premier siècle avant Jésus-Christ au deuxième siècle après) : 'Homarim lemilon - sidra aleph («Éléments pour un dictionnaire première série», Jérusalem, 1988). En plus de la concordance, elle a également publié les textes de ces sources, authentifiés par une comparaison scrupuleuse avec les meilleurs manuscrits connus pour chacune. Elle a publié enfin les listes lexicographiques afférentes : fréquence des mots, verbes et leurs séries paradigmatiques, substantifs et leurs formes déclinées, etc.

\subsubsection{MAAGARIM : le dictionnaire historique sur CD-ROM}

Lorsque la collecte du matériel sera achevée, l'équipe rédactionnelle souhaite proposer sur CD-ROM l'ensemble des témoignages réunis, permettant ainsi à toute personne intéressée d'étudier le matériel archivé et d'effectuer des recherches sous des angles divers : par entrées, par formes grammaticales, par combinaisons linguistiques, etc. Dès à présent, un premier CD-ROM de la série MAAGARIM est proposé au public. Il offre un corpus de 1750000 mots englobant la période ancienne (du second siècle avant JésusChrist au milieu du cinquième siècle après). En plus de la recherche d'une citation en partant d'une racine, d'un lemme ou d'une forme déclinée, dérivée ou combinée, on peut retrouver un texte spécifique à l'aide d'un titre, d'un nom d'auteur, d'une date ou du genre littéraire concerné ${ }^{2}$.

\subsubsection{Quelques exemples des résultats obtenus}

Les archives du «Programme de dictionnaire historique» ont déjà permis de retracer l'histoire précise des mots hébraïques figurant dans le corpus, jusqu'à la fin de l'époque des Tannaïm. C'est ainsi que, dans la Bible, on trouve le mot me'ate employé comme substantif (ex. : me'ate mayim/«un peu d'eau», Genèse XXIV,17), comme adverbe (ex. : Achab servit Baal me'atel «partiellement», II Rois X,18), et à deux reprises, comme adjectif («Ses jours seront me'atim/peu nombreux», Psaume 89,8). Ces emplois sont également attestés dans les rouleaux du désert de Judée (dont les Manuscrits de la mer Morte). Par contre, me'ate devient rare dans l'hébreu michnique, où il est employé exclusivement comme adverbe (six occurrences dans la concordance). En revanche, on le trouve attesté comme adjectif sous des formes diverses et déclinées : 51 fois sous les formes ma'oute, me'outa, me'outim (masculin et féminin singulier, masculin pluriel; ex. : Chènofo meroubè ouchorachav me'outine/«un arbre au feuillage abondant et aux racines rares», Avoth/Maximes des Pères, III,17). Toujours comme adjectif, on trouve également memou'at (masc. sing.), memou'atim (masc. pl.), etc. (50 occurrences, ex.: è'had meroubim vè̀'had memouatim/«l'un nombreux, l'autre rare», Berakhoth, 7,1). On trouve enfin la forme mou'ate (79 occurrences, ex. : mikra mou'ate vehalakhoth merouboth/«un petit verset et de nombreuses règles religieuses», 'Haguiga, 1,9). Et voici un autre exemple illustrant l'évolution de l'orthographe. Les mots $k a^{\prime}$ our, ou ki'our («laid», «laideur») ne s'écrivent avec un aleph intermédiaire que dans la Michna (7 occurrences; manuscrit Kaufmann). Partout ailleurs dans les sources recensées (Tossefta, Sifri, Mekhilta etc.), on le trouve orthographié avec un 'ayine intermédiaire (21 occurrences de ka'our, ke'oura, ki'our). Il est également orthographié avec un 'ayine dans le Livre de Ben-Sira (sous la forme : mekho'ar). Quant au verbe gama'/«boire», «avaler», on le trouve déjà dans la Bible, avec un aleph final, et il se décline comme tous les verbes défectifs dont la troisième consonne est un aleph ou un yod («consonnes faibles»). Il en va de même dans toute la littérature tannaïtique Michna, Sifri, Mekhilta, etc. (7 occurrences). Par contre, dans la Tossefta (15 occurrences), il est orthographié avec un 'ayine final (consonne forte), et on le trouve décliné comme les verbes réguliers. 
En conclusion, on peut dire qu'à l'heure actuelle, le programme de recherche du Dictionnaire historique de l'Académie de la langue hébraïque se concentre encore sur la collecte du matériel, son stockage dans une base de données informatisée, l'analyse grammaticale des mots et leur traitement sous forme de concordances. Lorsque le travail de rédaction du dictionnaire proprement dit pourra commencer, on ne négligera pas pour autant la collecte du matériel et la mise à jour du corpus, dont l'objectif est de servir de source permanente d'étude et de recherche.

Traduit de l'hébreu et complété par Francine Kaufmann.

\section{Notes}

1. Cf. Zeev Ben-'Haïm dans Lechonènou, vol. XXIII, 1959, Gan Ben-Ami Tsarfati, Lechonènou, vol. XXV, 1961 et de Merkin, Busharia et Meir dans Literary and Linguistic Computing, vol. 4, n 4, 1989, pp. 271-273. Cf. aussi Yevin, Lechonènou, vol. XL-XLI, 1989-1990, pp. 192-197 (numéro spécial à l'occasion du centenaire du Comité de la langue hébraïque). À titre de comparaison, notons que c'est au début des années 60 que l'Institut National de la Langue Française a entamé, à Nancy, l'élaboration d'un dictionnaire historique informatisé sur la langue du XIX ${ }^{\mathrm{e}}$ et du $\mathrm{XX}^{\mathrm{e}}$ siècle, depuis la Révolution française jusqu'à nos jours, dans le cadre du Trésor de la langue française. Cf. à ce sujet les articles de Yaakov Ben-Tolila dans Lechonènou, vol. XL, 1976, de Reouven Merkin dans LEXeter '83 Proceedings, R.R.K. Hartmann (Ed.), Tübingen, 1984, pp. 377-384, et dans Lechonènou, vol. L, 1986, pp. 126-129.

2. L'utilisation de MAAGARIM CD-ROM 1 exige un ordinateur compatible avec IBM 486 DX2, 66 MHz, 16 Mo de mémoire et 20 Mo d'espace libre sur le disque, écran couleur SVGA, lecteur CD-ROMx8; Windows 95, version hébraïque. Diffusion : The Academy of the Hebrew Language, POB 3449 Jérusalem 91034. Israel. (Tél. : 972-2-5632242. Fax : 972-2-5617065. Email : acad2u@vms.huji.ac.il). 